TITLE:

\title{
Pump-probe measurement of ultrafast all-optical modulation based on intersubband transition in n-doped quantum wells
}

$\operatorname{AUTHOR}(S):$

Asano, T; Tamura, M; Yoshizawa, S; Noda, S

\section{CITATION:}

Asano, T ... [et al]. Pump-probe measurement of ultrafast all-optical modulation based on intersubband transition in n-doped quantum wells. APPLIED PHYSICS LETTERS 2000, 77(1): 19-21

ISSUE DATE:

2000-07-03

URL:

http://hdl.handle.net/2433/50161

\section{RIGHT:}

Copyright 2000 American Institute of Physics. This article may be downloaded for personal use only. Any other use requires prior permission of the author and the American Institute of Physics. 


\title{
Pump-probe measurement of ultrafast all-optical modulation based on intersubband transition in $\boldsymbol{n}$-doped quantum wells
}

\author{
T. Asano, ${ }^{\text {a) }}$ M. Tamura, S. Yoshizawa, and S. Noda \\ Department of Electrical Science and Engineering, Kyoto University, Kyoto 606-8317, Japan
}

(Received 28 October 1999; accepted for publication 11 May 2000)

\begin{abstract}
Modulation of interband-resonant light $(\sim 800 \mathrm{~nm})$ by intersubband-resonant light $(5-7 \mu \mathrm{m})$ was investigated in $n$-doped AlGaAs/GaAs multiple quantum wells by a two-color femtosecond pump-probe technique. Modulation with a recovery time of $\sim 1$ ps is observed in a plainer-type modulation device at room temperature. The modulation of interband absorption coefficient is $\sim 1000 \mathrm{~cm}^{-1}$ when the energy density of the intersubband light pulse is $\sim 4 \mathrm{fJ} / \mu \mathrm{m}^{2}$. The modulation efficiency indicates that $99 \%$ modulation can be achieved with a control pulse energy of $\sim 1 \mathrm{pJ}$ when a conventional waveguide-type device structure is utilized. The mechanism which determines the modulation speed is discussed in terms of carrier relaxation process. It is shown that the modulation speed is mainly determined by the inter- and intrasubband relaxation times, where the latter is influenced by hot phonon effects. (C) 2000 American Institute of Physics.

[S0003-6951(00)02727-3]
\end{abstract}

The intersubband transition (ISB-T) in quantum well (QW) structures has interesting properties such as a large transition dipole moment in the midinfrared range ${ }^{1}$ and an ultrafast energy relaxation time due to longitudinal optical (LO) phonon scattering ( picoseconds $)^{2,3}$ The ultrafast relaxation process of the ISB-T is important for basic physics as well as for the development of high-speed optical devices. The authors previously proposed an ultrafast all-optical modulation scheme that utilizes the ISB-T. ${ }^{4}$ This scheme uses three subbands formed in a $n$-doped QW (Fig. 1): the first valence subband (VB1) and the first and second conduction subbands (CB1 and $\mathrm{CB} 2)$. Interband (IB) resonant light absorption ( $\mathrm{VB} 1 \rightarrow \mathrm{CB} 1$ ) can be increased by exciting the electrons from $\mathrm{CB} 1$ to $\mathrm{CB} 2$ using ISB-resonant light. The increased absorption decreases as the electrons relax from $\mathrm{CB} 2$ to $\mathrm{CB} 1$, and the relaxation time appears to be as fast as $\sim 1$ ps. Thus, it is thought that an IB-resonant light signal can be modulated using an ISB-resonant light signal on the order of picoseconds.

Theoretical and experimental investigations have revealed the static (not ultrafast) characteristics of the modulation. ${ }^{4-7}$ However, the modulation speed was not determined in the previous reports. In this letter, we report femtosecond time-resolved measurement results for the alloptical modulation. A planar-type GaAs/AlGaAs QW modulation device was fabricated and investigated using a twocolor pump-probe technique. The modulation speed is shown to be $\sim 1.3 \mathrm{ps}$. The relaxation process of the ISB-excited electrons is also discussed.

The modulation device investigated consists of 150 periods $\left(N_{\text {well }}\right)$ of $\operatorname{GaAs}(59 \AA) / \mathrm{Al}_{0.35} \mathrm{Ga}_{0.65} \mathrm{As}(150 \AA)$ multiple QWs grown on a semi-insulating GaAs substrate by molecular beam epitaxy. The barrier layer was selectively doped at a Si density of $1.5 \times 10^{18} \mathrm{~cm}^{-3}$. A 0.5 - $\mu \mathrm{m}$-thick $\mathrm{Al}_{0.4} \mathrm{Ga}_{0.6} \mathrm{As}$ etch stopping layer was grown between the QW layer and the

${ }^{a)}$ Electronic mail: tasano@kuee.kyoto-u.ac.jp
GaAs substrate. The device was fabricated by fixing the sample on a thin transparent plate, and the GaAs substrate was removed by selective wet etching in order to make the device transparent to IB-resonant light. The midinfrared absorption spectrum of the QWs was measured in the conventional Brewster angle configuration by Fourier-transform infrared spectroscopy (FTIR) [Fig. 3(b)]. An optical absorption peak due to the ISB-T was observed at $7.2 \mu \mathrm{m}(172 \mathrm{meV})$. The absorption peak was $\sim 19 \%$, and the full width at half maximum (FWHM) was $\sim 20 \mathrm{meV}$. The near-infrared absorption spectrum of the sample was also measured and the IB absorption edge (VB1 $\rightarrow$ CB1) was found to be about $830-840 \mathrm{~nm}$.

Time-resolved modulation experiments were carried out using the two-color pump-probe method, in which the device is pumped by a midinfrared $(4-9 \mu \mathrm{m})$ control light pulse and is probed by a near-infrared $(\sim 800 \mathrm{~nm})$ signal light pulse. The pump pulse was generated by optical parametric amplification and differential frequency mixing using an $800 \mathrm{~nm}$ mode-locked Ti-sapphire laser pulse. The residual of the $800 \mathrm{~nm}$ light pulse was used for the probe. The temporal and spectral width of the pump and probe pulses were $\sim 120 \mathrm{fs}$ and $\sim 20 \mathrm{meV}$, respectively. The pump beam was incident to

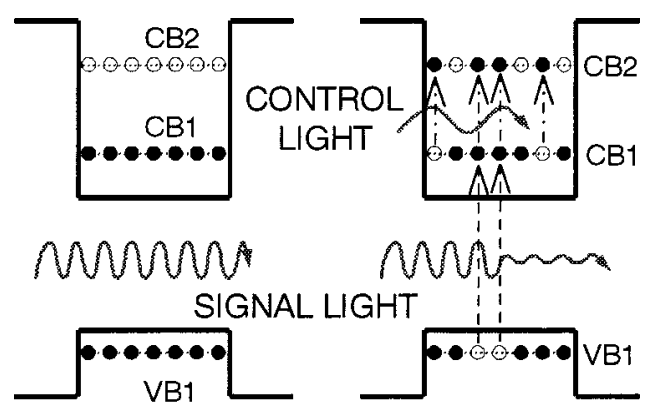

(a)

(b)

FIG. 1. Schematic diagram of the all-optical-modulation scheme in a n-doped QW (a) without and (b) with control light. 


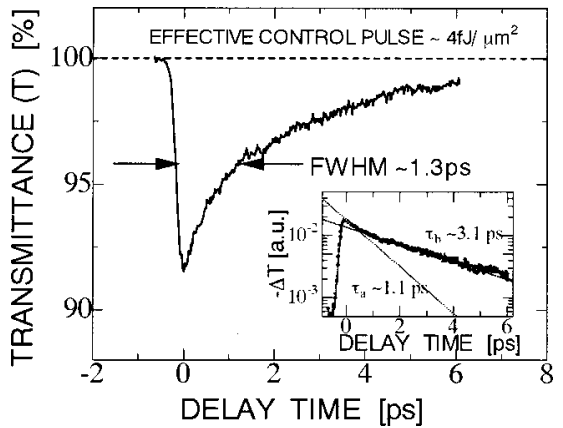

FIG. 2. Example of time-resolved all-optical-modulation characteristics. Inset is a logarithmic plot of the modulation depth.

the sample at the Brewster angle in order to ensure sufficient interaction with the ISB dipole moment and also to suppress multiple reflections inside the sample. The incident angle for the probe beam was almost the same as that of the pump beam. The effective energy density of the control pulse $\left(J_{c}\right)$, which is actually absorbed by the QWs, was varied from $\sim 2$ to $\sim 140 \mathrm{fJ} / \mu \mathrm{m}^{2}$. A sufficiently small energy density of $<\sim 5$ $\mathrm{fJ} / \mu \mathrm{m}^{2}$ was used for the signal pulse so as not to supply too many electrons from VB1 to CB1.

Figure 2 shows the temporal trace of the change in the transmittance of the probe (signal) pulse induced by the pump (control) pulse (i.e., the modulation profile), where $J_{c}$ was $\sim 4 \mathrm{fJ} / \mu \mathrm{m}^{2}$ and the center wavelength of the control pulse $\left(\lambda_{c}\right)$ was tuned to the ISB-resonance $(7.2 \mu \mathrm{m})$. The rapid decrease in the transmittance and its ultrafast recovery can be clearly seen in the figure. The recovery time of the modulation (measured as FWHM of the modulation profile) is as short as $1.3 \mathrm{ps}$. We also carried out the measurements while gradually detuning $\lambda_{c}$ from the ISB resonance. Figure 3 shows the peak of the modulation depth measured as a function of $\lambda_{c}$ together with the ISB absorption spectrum of the QWs. The correlation between the modulation and absorption spectra clearly demonstrates that the observed modulation is due to the ISB-T.

The peak modulation depth observed in the first measurement (Fig. 2) corresponds to the increase in the absorption coefficient of as much as $\sim 1000 \mathrm{~cm}^{-1}$, where the energy density of the control pulse was $\sim 4 \mathrm{fJ} / \mu \mathrm{m}^{2}$. It is estimated that $\sim 99 \%$ modulation of the signal light can be

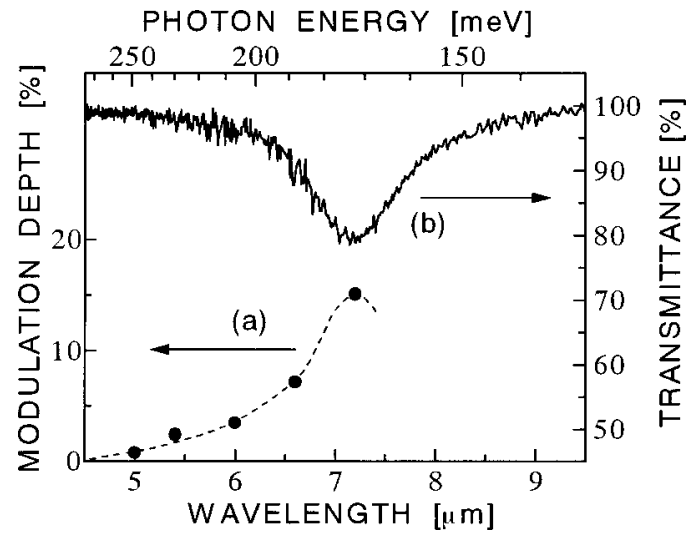

FIG. 3. (a) Peak modulation depth measured as a function of the control light wavelength (data for the longer wavelength side could not be measured due to limitations in the light source), and (b) infrared absorption spectrum

of the sample measured by FTIR.
Downloaded 04 Mar 2008 to 130.54.110.22. Redistribution subject to AIP license or copyright; see http://apl.aip.org/apl/copyright.jsp

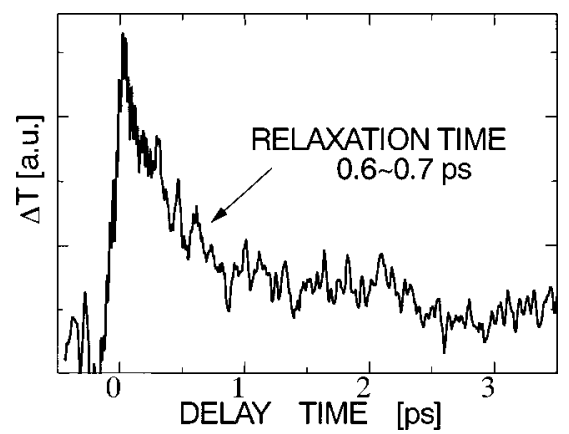

FIG. 4. Relaxation curve of ISB absorption saturation measured by onecolor pump-probe method. The sample was pumped and also probed by ISB-resonant light pulses $(\sim 7.2 \mu \mathrm{m})$.

obtained with a control pulse energy of $\sim 1 \mathrm{pJ}$ when a conventional waveguide-type device configuration, which can enhance the interaction between QWs and light, is used instead of the surface incident configuration used here. The relatively high modulation efficiency is due to the fact that the transition dipole moment of the ISB-T in the midinfrared range is very large $(\sim 17 \mathrm{e} \AA$ in this $\mathrm{QW})$ and the nearinfrared photon $(\sim 1.5 \mathrm{eV})$ is controlled by the midinfrared photon $(\sim 0.17 \mathrm{eV})$.

The inset in Fig. 2 shows a logarithmic plot of the modulation profile. The modulation decays with a short relaxation time of $\sim 1.1 \mathrm{ps}\left(T_{a}\right)$ soon after the ISB-excitation (delay time $0 \sim 1 \mathrm{ps}$ ), and subsequently the relaxation time becomes longer $\left(\sim 3 \mathrm{ps}: T_{b}\right)$. Furthermore, we measured the recovery time of ISB absorption saturation in the same QWs, where the QWs were pumped and probed by the ISB-resonant light pulses (Fig. 4). It can be seen in Fig. 4 that the ISB absorption recovery time $\left(T_{\text {isb }}\right)$ is $0.6-0.7 \mathrm{ps}$. The discrepancy between $T_{a}$ and $T_{\text {isb }}$ can be explained in terms of the relaxation process. In the latter case, the pump-induced absorption saturation recovers when the excited electrons are scattered from CB2 to the higher energy part of CB1 since the two subbands are almost parallel [Fig. 5(a)] However, in the former case, the electrons must relax from the higher energy part of CB1 to the bottom of CB1 (intrasubband energy relaxation) in order to reduce the IB absorption near the band edge [Fig. $5(b)]$. The intrasubband energy relaxation process is expected to take an additional $0.3-0.4$ ps since the ISB energy spacing $\left(E_{\mathrm{isb}} \sim 172 \mathrm{meV}\right)$ is about $4-5$ times that of the LO

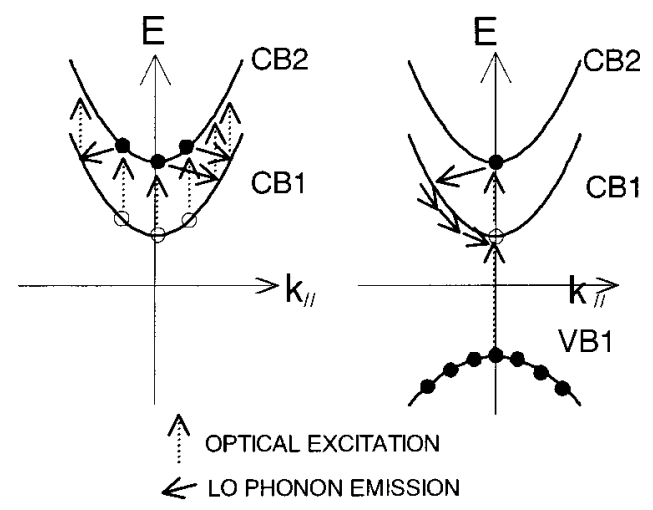

(a)

(b)
FIG. 5. Schematic diagram showing the electron relaxation process for reAlP license or copyright; see http://apl.aip.org/apl/copyright.jsp 
phonon energy and each LO phonon emission takes $\sim 0.1$ ps. ${ }^{3}$ (Electron-electron scattering is much faster, but it is not a energy relaxation process). Therefore, the discrepancy between $T_{\text {isb }}(0.6-0.7 \mathrm{ps})$ and $T_{a}(\sim 1.1 \mathrm{ps})$ can be explained by the additional intrasubband energy relaxation time $(0.3-0.4$ ps). We assumed that the long relaxation time observed in the subsequent decay process $\left(T_{b}: \sim 3 \mathrm{ps}\right)$ is due to the reabsorption of hot phonons emitted during intrasubband relaxation. ${ }^{8,9}$ (The hot phonons emitted by the ISB scattering have no effect on intrasubband phonon scattering due to the different symmetry.) Then, we estimated the hot LO population (number of phonons per mode) that is generated by the complete intrasubband energy relaxation for the case in the present experiment. The total number of hot LO phonons $\left(n_{p}\right)$ can be estimated from $J_{c}$ by the equation ${ }^{10}$

$$
n_{p}=\frac{J_{c} / E_{\mathrm{isb}}}{N_{\mathrm{well}}}\left(E_{\mathrm{isb}} / \hbar \omega_{\mathrm{LO}}-1\right) .
$$

The number of LO phonon modes that contribute to the intrasubband relaxation $\left(N_{p}\right)$ can now be estimated by the following equation: ${ }^{3}$

$$
N_{p}=\frac{2 q_{\| \min }^{2}}{\pi} N_{\perp},
$$

where $q_{\mathrm{min}}=\sqrt{2 m^{*}\left(E_{\mathrm{isb}}-\hbar \omega_{\mathrm{LO}}\right)} / \hbar-\sqrt{2 m^{*}\left(E_{\mathrm{isb}}-2 \hbar \omega_{\mathrm{LO}}\right)} /$ $\hbar$ is the minimum in-plane momentum $\left(q_{\|}\right)$of the LO phonon which is emitted during the intrasubband relaxation process. $^{3}$ In the equation, we cut off the phonon modes whose $q_{\|}$is greater than $3 q_{\| \min }$ since the electron-LO phonon interaction strength is proportional to $1 / q_{\|}^{2} . N_{\perp}$ is the number of vertical modes of the LO phonon. We assumed that two vertical phonon modes (the first mode of a GaAs-wellconfined phonon and the symmetric mode of a AlAsinterface-trapped phonon) are the main contributors to the relaxation. ${ }^{3}$ The average hot phonon population $\left(n_{\mathrm{av}}\right)$ can be calculated from the equation $n_{\mathrm{av}}=n_{p} / N_{P}$. By using these equations and conventional material parameters, $n_{\mathrm{av}}$ is estimated to be $\sim 0.2$ in the case of the experiment shown in Fig. 2 , which is as large as the LO phonon population in thermal equilibrium at $300 \mathrm{~K}$. Since the relaxation time of hot LO phonons is as long as $5-7 \mathrm{ps},{ }^{11,12}$ it is possible that electron intraband relaxation becomes slower by an order of picoseconds as a result of the hot phonon reabsorption process. Moreover, in additional experiments, it was observed that $T_{b}$ becomes longer from $\sim 3$ to $\sim 7$ ps as $J_{c}$ increases from $\sim 2$ to $\sim 140 \mathrm{fJ} / \mu \mathrm{m}^{2}$. The results suggest that $T_{b}$ is influenced by the effects of hot phonon reabsorption. Therefore, utilization of a waveguide-type configuration with a low energy density control pulse is preferable since hot phonon effects can be avoided.

In conclusion, we carried out time-resolved measurement of an all-optical modulation scheme that utilizes the ISB-T by means of a two-color pump probe technique. It was directly shown that ultrafast operation in the range of $1.3 \mathrm{ps}$ (FWHM) is possible in this scheme using a relatively low control light pulse of $4 \mathrm{fJ} / \mu \mathrm{m}^{2}$. The results indicate that the ISB-T is useful for realizing ultrafast low-energy all-optical modulation and switching.

This work was supported in part by a Grant-in-Aid for Scientific Research from the Ministry of Education, Science, Sports and Culture of Japan, the Femtosecond Technology Research Association (FESTA) which is supported by New Energy and Industrial Technology Development Organization (NEDO), and VBL Kyoto University.

${ }^{1}$ L. C. West and S. J. Eglash, Appl. Phys. Lett. 46, 1156 (1985).

${ }^{2}$ K. Huan and B. Zhu, Phys. Rev. B 38, 13377 (1988).

${ }^{3}$ H. Rücker, E. Molinari, and P. Lugli, Phys. Rev. B 45, 6747 (1992).

${ }^{4}$ S. Noda, T. Uemura, T. Yamashita, and A. Sasaki, J. Appl. Phys. 68, 6529 (1990)

${ }^{5}$ S. Noda, M. Ohya, Y. Muromoto, T. Asano, and A. Sasaki, IEEE J. Quantum Electron. 31, 1683 (1995).

${ }^{6}$ T. Suzuki, T. Mitsuyu, K. Nishi, H. Ohyama, T. Tomimasu, S. Noda, T. Asano, and A. Sasaki, Appl. Phys. Lett. 69, 4136 (1996).

${ }^{7}$ T. Asano, S. Noda, A. Sasaki, T. Suzuki, T. Mitsuyu, K. Nishi, H. Ohyama, and T. Tomimasu, Nucl. Instrum. Methods Phys. Res. B 144, 123 (1998).

${ }^{8}$ H. Lobentanzer, W. Stolz, J. Nagle, and K. Ploog, Phys. Rev. B 39, 5234 (1989).

${ }^{9}$ C. Y. Tsai, L. F. Eastman, and Y. H. Lo, Appl. Phys. Lett. 63, 3408 (1993).

${ }^{10}$ T. Asano, S. Noda, and K. Tomoda, Appl. Phys. Lett. 74, 1418 (1999).

${ }^{11}$ D. Linde, J. Kuhl, and H. Klingenberg, Phys. Rev. Lett. 44, 1505 (1980).

${ }^{12}$ M. C. Tathan, J. F. Ryan, and C. T. Foxton, Phys. Rev. Lett. 63, 1637 (1989). 